\title{
THE MOORE-PENROSE INVERSE OF A SUM OF MATRICES
}

\author{
CHING-HSIANG HUNG and THOMAS L. MARKHAM
}

(Received 3 June 1975)

\begin{abstract}
Suppose $U$ and $V$ are $m \times n$ matrices over the complex field. We obtain a representation for the Moore-Penrose inverse of the sum $U+V$. A well-known result of Cline is then derived as a special case of a corollary of this representation.
\end{abstract}

\section{Introduction}

If $A$ is an $m \times n$ matrix over the complex field, then the Moore-Penrose inverse of $A$, which is denoted by $A^{+}$, is an $n \times m$ matrix such that

$$
\begin{aligned}
A A^{+} A & =A \\
A^{+} A A^{+} & =A^{+} \\
\left(A A^{+}\right)^{*} & =A A^{+} \\
\left(A^{+} A\right)^{*} & =A^{+} A .
\end{aligned}
$$

Suppose $U, V$ are $m \times n$ matrices. Cline (1965) obtained a formula for $(U+V)^{+}$under the assumption $U V^{*}=0$. We will derive a formula for $(U+V)^{+}$by constructing a new matrix from $U, V, J_{n}$, and $J_{m}$ where

$$
J_{k}=\left(j_{s s}\right)_{k \times k}, \quad j_{t s}=\left\{\begin{array}{l}
1 \text { if } s=k-t+1 \\
0 \text { otherwise }
\end{array}\right.
$$

and using our result in Hung, Markham (1975). We note at this point that $J_{k}$ is that permutation matrix with ones on the secondary diagonal and zeroes elsewhere, and $J_{k}^{2}=I$.

Furthermore, we determine necessary and sufficient conditions for 385

(C) Copyright Australian Mathematical Society 1977

Copyright. Apart from any fair dealing for scholurly purposes as permitted under the Copyright Act, no part of this JOURNAL may be reproduced by any process without written permission from the Treasurer of the Australian Mathernatical Society. 
$(U+V)^{+}$to have the form stated in Cline's Theorem 2, p. 106. We then give some simpler forms for certain special cases. Finally we give an example for which $U^{*} V \neq 0$ but $(U+V)^{+}$has the form given in Cline (1965).

Some techniques employed in the proofs of our theorems are similar to techniques used in Pye, Boullion, and Atchison (1973).

\section{A formula for $(U+V)^{+}$}

THEOREM 1. If both $U$ and $V$ are $m \times n$ matrices, then

$$
(U+V)^{+}=J_{n} J^{-1}\left[\left(I-T^{*} K^{+} E\right) L^{+}\left(J_{n}-E^{*} K^{+}\right)+T^{*} K^{+}\right]\left(U^{*}+V^{*}\right),
$$

where $J_{n}$ is as defined in (1.5), and

$$
\begin{aligned}
K & =U^{*} U+V^{*} V, \\
E & =\left(U^{*} V+V^{*} U\right) J_{n}, \\
R & =V J_{n}-U K^{+} E, \\
S & =J_{m} U J_{n}-J_{m} V K^{+} E, \\
L & =R^{*} R+S^{*} S, \\
T & =K^{+} E\left(I-L^{+} L\right), \\
J & =I+T^{*} T .
\end{aligned}
$$

Proof. Let

$$
Q_{1}=\frac{1}{\sqrt{2}}\left(\begin{array}{cc}
I & -J_{m} \\
J_{m} & I
\end{array}\right), \quad Q_{2}=\frac{1}{\sqrt{2}}\left(\begin{array}{cc}
I & -J_{n} \\
J_{n} & I
\end{array}\right)
$$

and

$$
M=\left(\begin{array}{cc}
U & V J_{n} \\
J_{m} V & J_{m} U J_{n}
\end{array}\right)
$$

Then,

$$
\begin{aligned}
N & =Q_{1}^{T} M Q_{2}=\frac{1}{2}\left(\begin{array}{cc}
2(U+V) & 0 \\
0 & 2 J_{m}(U-V) J_{n}
\end{array}\right) \\
& =\operatorname{diag}\left((U+V), J_{m}(U-V) J_{n}\right) .
\end{aligned}
$$

Thus, $N^{+}=\operatorname{diag}\left((U+V)^{+}, J_{n}(U-V)^{+} J_{m}\right)$. Therefore, $M=Q_{1} N Q_{2}^{T}$ implies $M^{+}=Q_{2} N^{+} Q_{1}^{r}$. That is,

$$
M^{+}=\frac{1}{2}\left(\begin{array}{cc}
(U+V)^{+}+(U-V)^{+} & {\left[(U+V)^{+}-(U-V)^{+}\right] J_{m}} \\
J_{n}\left[(U+V)^{+}-(U-V)^{+}\right] & J_{n}\left[(U+V)^{+}+(U-V)^{+}\right] J_{m}
\end{array}\right) .
$$


Now, by the theorem in Hung, Markham (1975), we have

$$
M^{+}=\left(\begin{array}{cc}
K^{+}\left(U^{*}-E F\right) & K^{+}\left(V^{*} J_{m}-E H\right) \\
F & H
\end{array}\right),
$$

where

$$
\begin{aligned}
K & =U^{*} U+V^{*} V, \\
E & =\left(U^{*} V+V^{*} U\right) J_{n}, \\
R & =V J_{n}-U K^{+} E, \\
S & =J_{m} U J_{n}-J_{m} V K^{+} E, \\
L & =R^{*} R+S^{*} S, \\
T & =K^{+} E\left(I-L^{+} L\right), \\
J & =I+T^{*} T, \\
F & =L^{-} R^{*}+J^{-1} T^{*} K^{+}\left(U^{*}-E L^{+} R^{*}\right), \\
H & =L^{+} S^{*}+J^{-1} T^{*} K^{+}\left(V^{*} J_{m}-E L^{+} S^{*}\right) .
\end{aligned}
$$

Since the Moore-Penrose inverse is unique, we have

$$
\begin{gathered}
\frac{1}{2}\left[(U+V)^{+}+(U-V)^{+}\right]=K^{+}\left(U^{*}-E F\right), \\
\frac{1}{2}\left[(U+V)^{+}-(U-V)^{+}\right] J_{m}=K^{+}\left(V^{*} J_{m}-E H\right), \\
\frac{1}{2} J_{n}\left[(U+V)^{+}-(U-V)^{+}\right]=F, \\
\frac{1}{2} J_{n}\left[(U+V)^{+}+(U-V)^{+}\right] J_{m}=H .
\end{gathered}
$$

Thus, from (2.1) and (2.2), we get

$$
(U+V)^{+}=K^{+}\left(U^{*}+V^{*}\right)-K^{+} E\left(F+H J_{m}\right)
$$

and from (2.3) and (2.4), we obtain

$$
(U+V)^{+}=J_{n} F+J_{n} H J_{m}
$$

Now, $S^{*} J_{m}+R^{*}=\left(J_{n}-E^{*} K^{+}\right)\left(U^{*}+V^{*}\right)$ implies

$$
J_{n} F+J_{n} H J_{m}=J_{n} J^{-1}\left[\left(I-T^{*} K^{+} E\right) L^{+}\left(J_{n}-E^{*} K^{+}\right)+T^{*} K^{+}\right]\left(U^{*}+V^{*}\right) .
$$

Hence the proof is complete.

Cline (1965) used the condition $U V^{*}=0$ and his formula for $\left(U U^{*}+V V^{*}\right)^{+}$to obtain a formula for $(U+V)^{+}$. From our theorem, we obtain the following corollary, of which Cline's formula is a special case. Cline's condition $U V^{*}=0$ becomes $U^{*} V=0$ in our corollary, and this implies $(U+V) E=(U+V)\left(U^{*} V+V^{*} U\right) J_{n}=0$ which is a necessary and sufficient condition for $(U+V)^{+}$to have the form (3.1) of Cline (1965). 
Let $N(A)$ denote the null column space of $A$. It is well-known that $N(A) \subset N(B)$ if and only if $B=B A^{+} A$.

Corollary 1.1. $(U+V)^{+}=K^{+}\left(U^{*}+V^{*}\right)$ if and only if $(U+V) E=$ 0 , and in this case, we have

$K^{+}\left(U^{*}+V^{*}\right)=U^{+}+\left[G^{*+}+\left(I-G^{*+} V\right) U^{+} U^{*+} V^{*} Q^{*}\left(I-G^{+} G\right)\right]\left(I-V U^{+}\right)$, where $G=\left(I-U^{*} U^{*+}\right) V^{*}$, and

$$
Q=\left[I+\left(I-G^{+} G\right) V U^{+} U^{*+} V^{*}\left(I-G^{+} G\right)\right]^{-1} .
$$

Proof. Suppose $(U+V)^{+}=K^{+}\left(U^{*}+V^{*}\right)$. Then, $(U+V)(U+V)^{+}$ $(U+V)=(U+V) K^{+}\left(K+E J_{n}\right)$. This implies $(U+V)=(U+V)+$ $(U+V) K^{+} E J_{n} \quad$ since $\quad N(K) \subset N(U) \quad$ and $\quad N(K) \subset N(V)$. Thus, $(U+V) K^{+} E J_{n}=0$. Hence, $(U+V) K^{+} E=0$ since $J_{n}^{2}=I$. Therefore, $E^{*} K^{+}\left(U^{*}+V^{*}\right)=0$. Now by assumption $(U+V)^{+}=K^{+}\left(U^{*}+V^{*}\right)$, we obtain $E^{*}(U+V)^{+}=0$. This implies $(U+V) E=0$ since $B A^{+}=0$ if and only if $A B^{*}=0$. On the other hand, if $(U+V) E=0$, then $E^{*}(U+V)^{+}=0$. Thus, by (2.6) we have $E^{*}\left(J_{n} F+J_{n} H J_{m}\right)=0$. This implies $J_{n} E^{*} J_{n}\left(F+H J_{m}\right)=0$. Hence, $E\left(F+H J_{m}\right)=0$ since $J_{n} E^{*} J_{n}=E$. Therefore, by (2.5), we have $(U+V)^{+}=K^{+}\left(U^{*}+V^{*}\right)$. This completes the proof.

Corollary 1.2. $(U+V)^{+}=J_{n} L^{+}\left(J_{n}-E^{*} K^{+}\right)\left(U^{*}+V^{*}\right)$ if and only if $N(L) \subset N\left((U+V) J_{n}\right)$.

Proof. Suppose $(U+V)^{+}=J_{n} L^{+}\left(J_{n}-E^{*} K^{+}\right)\left(U^{*}+V^{*}\right)$. Then,

$$
(U+V)^{+}=J_{n} L^{+}\left(S^{*} J_{m}+R^{*}\right)=J_{n} L^{+}\left(L H J_{m}+L F\right)=J_{n} L^{+} L J_{n}(U+V)^{+} .
$$

This implies $\left(I-J_{n} L^{+} L J_{n}\right)(U+V)^{+}=0$. Thus, $(U+V)\left(I-J_{n} L^{+} L J_{n}\right)=0$. That is, $(U+V)=(U+V) J_{n} L^{+} L J_{n}$ or $(U+V) J_{n}=(U+V) J_{n} L^{+} L$. Hence, $N(L) \subset N\left((U+V) J_{n}\right)$. follows.

For the necessity, we reverse the proof for sufficiency. Then the result

Corollary 1.3. $(U+V)^{+}=J_{n} J^{-1} T^{*} K^{+}\left(U^{*}+V^{*}\right)$ if and only if $L=0$.

Proof. Suppose $(U+V)^{+}=J_{n} J^{-1} T^{*} K^{+}\left(U^{*}+V^{*}\right)$. Then, by Theorem 1, we can get $J_{n} J^{-1}\left[\left(I-T^{*} K^{+} E\right) L^{+}\left(J_{n}-E^{*} K^{+}\right)+T^{*} K^{+}\right]\left(U^{*}+V^{*}\right)$ $=J_{n} J^{-1} T^{*} K^{+}\left(U^{*}+V^{*}\right)$.

This implies

$$
\left(I-T^{*} K^{+} E\right) L^{+}\left(J_{n}-E^{*} K^{+}\right)\left(U^{*}+V^{*}\right)=0 .
$$

Postmultiplying $(2.7)$ by $(U+V) J_{n}$, we obtain 


$$
\left(I-T^{*} K^{+} E\right) L^{+}\left(J_{n}-E^{*} K^{+}\right)\left(U^{*}+V^{*}\right)(U+V) J_{n}=0 .
$$

Premultiplying (2.8) by $L$ and using the fact $L T^{*}=0$, we have

$$
L L^{+}\left(J_{n}-E^{*} K^{+}\right)\left(K+\dot{E} J_{n}\right) J_{n}=0 .
$$

That is,

$$
L L^{+}\left(J_{n} K-E^{*} K^{+} K+J_{n} E J_{n}-E^{*} K^{+} E J_{n}\right) J_{n}=0 .
$$

Thus, $L L^{+}\left(J_{n} K J_{n}-E^{*} K^{+} E\right)=0$ since $N(K) \subset N\left(E^{*}\right)$ and $E^{*}=J_{n} E J_{n}$. Therefore, $L L^{+} L=0$ since $L=J_{n} K J_{n}-E^{*} K^{+} E$. This implies $L=0$. On the other hand, if $L=0$, then the result follows immediately from Theorem 1 .

If we let $P=I+T T^{*}$, then the formula for

$$
\left(\begin{array}{cc}
U & V J_{n} \\
J_{m} V & J_{m} U J_{n}
\end{array}\right)
$$

can be written as

$$
M^{+}=\left(\begin{array}{cc}
P^{-1} K^{+}\left(U^{*}-E L^{+} R^{*}\right) & P^{-1} K^{+}\left(V^{*} J_{m}-E L^{+} S^{*}\right) \\
L^{+} R^{*}+T^{*} P^{-1}\left(U^{*}-E L^{+} R^{*}\right) & L^{+} S^{*}+T^{*} P^{-1}\left(V^{*} J_{m}-E L^{+} S^{*}\right)
\end{array}\right) .
$$

Thus, by the uniqueness of $M^{+}$, we have

$$
\begin{gathered}
\frac{1}{2}\left((U+V)^{+}+(U-V)^{+}\right)=P^{-1} K^{+}\left(U^{*}-E L^{+} R^{*}\right) \\
\frac{1}{2}\left((U+V)^{+}-(U-V)^{+}\right) J_{m}=P^{-1} K^{+}\left(V^{*} J_{m}-E L^{+} S^{*}\right) .
\end{gathered}
$$

Thus, from (2.9) and (2.10), we get

$$
(U+V)^{+}=P^{-1} K^{+}\left(U^{*}+V^{*}\right)-P^{-1} K^{+} E L^{+}\left(R^{*}+S^{*} J_{m}\right) .
$$

This implies

$$
(U+V)^{+}=P^{-1} K^{+}\left(I-E L^{+}\left(J_{n}-E^{*} K^{+}\right)\right)\left(U^{*}+V^{*}\right)
$$

since $R^{*}+S^{*} J_{m}=\left(J_{n}-E^{*} K^{+}\right)\left(U^{*}+V^{*}\right)$. Hence we have obtained an alternate representation of $(U+V)^{+}$.

THEOREM 2. If both $U$ and $V$ are $m \times n$ matrices, then

$$
(U+V)^{+}=P^{-1} K^{+}\left(I-E L^{+}\left(J_{n}-E^{*} K^{+}\right)\right)\left(U^{*}+V^{*}\right) .
$$

Corollary 2.1. $(U+V)^{+}=P^{-1} K^{+}\left(U^{*}+V^{*}\right)$ if and only if $R E^{*}=0$ and $S E^{*}=0$.

Proof. Suppose $(U+V)^{+}=P^{-1} K^{+}\left(U^{*}+V^{*}\right)$. Then, from (2.11), we have 


$$
P^{-1} K^{+} E L^{+}\left(J_{n}-E^{*} K^{+}\right)\left(U^{*}+V^{*}\right)=0 .
$$

Premultiplying (2.12) by $P$ and then postmultiplying by $(U+V) J_{n}$, we get $K^{+} E L^{+}\left(J_{n}-E^{*} K^{+}\right)\left(K+E J_{n}\right) J_{n}=0$. This implies $E L^{+} L=0$ since $N(K) \subset N\left(E^{*}\right), E^{*}=J_{n} E J_{n}$, and $L=J_{n} K J_{n}-E^{*} K^{+} E$, as in the proof of Corollary 1.3. Hence $E L^{+}=0$. This implies $E L=0$ since $L^{+} L=L L^{+}$. Now $L=R^{*} R+S^{*} S$, so we have $E R^{*} R E^{*}+E S^{*} S E^{*}=0$. This implies $R E^{*}=0$ and $S E^{*}=0$. On the other hand if $R E^{*}=0$ and $S E^{*}=0$, then $R^{*} R E^{*}+S^{*} S E^{*}=0$, which implies $L E^{*}=0$. That is $E L=0$. Therefore $E L^{+}=0$ and the result follows by (2.11).

Corollary 2.2. $(U+V)^{+}=K^{+}\left[I-E L^{+}\left(J_{n}-E^{*} K^{+}\right)\right] \quad\left(U^{*}+V^{*}\right)$ if and only if $(U+V) T=0$.

Proof. Suppose

$$
(U+V)^{+}=K^{+}\left(I-E L^{+}\left(J_{n}-E^{*} K^{+}\right)\right)\left(U^{*}+V^{*}\right) .
$$

Postmultiplying (2.13) by $(U+V) J_{n}$ and premultiplying by $(U+V)$, we get

$$
(U+V) J_{n}=(U+V) K^{+}\left(\left(K+E J_{n}\right) J_{n}-E L^{+} L\right) .
$$

Thus $(U+V) K^{+} E\left(I-L^{+} L\right)=0$ since $N(K) \subset N(U)$ and $N(K) \subset N(V)$. Hence $(U+V) T=0$ since $T=K^{+} E\left(I-L^{+} L\right)$. On the other hand if $(U+V) T=0$, then

$$
T^{*}\left(U^{*}+V^{*}\right)=0 .
$$

Postmultiplying (2.14) by $\left((U+V)(U+V)^{*}\right)^{+}$, we have $T^{*}(U+V)^{+}=0$. This implies $T T^{*}(U+V)^{+}=0$. Therefore, $P(U+V)^{+}=(U+V)^{+}$since $P=$ $I+T T^{*}$. From Theorem 2, we can see that

$$
P(U+V)=K^{+}\left(I-E L^{+}\left(J_{n}-E^{*} K^{+}\right)\right)\left(U^{*}+V^{*}\right) .
$$

Hence the proof is complete. Furthermore, we have noted the following:

$$
(U+V) T=0 \text { if and only if } N(L) \subset N\left((U+V) K^{+} E\right)
$$

and in particular if $L$ is nonsingular, then $T=0$ implies $(U+V) T=0$. Therefore Corollary 2.2 is applicable if $L$ is nonsingular. In case $L=0$, the conditions of Corollary 2.1 are satisfied.

\section{An application}

We can use Theorem 1 to determine $(A B)^{+}$. If we partition

$$
A=\left(A_{1}, A_{2}\right), \quad B=\left(\begin{array}{l}
B_{1} \\
B_{2}
\end{array}\right)
$$


conformably, then $A B=A_{1} B_{1}+A_{2} B_{2}$, so now we can apply the theorem with $U=A_{1} B_{1}, V=A_{2} B_{2}$. For example, suppose

$$
\begin{aligned}
& A=\left(\begin{array}{rr|rrr}
1 & 0 & 0 & 0 & 0 \\
0 & 1 & 0 & 1 & 0 \\
-1 & 0 & 1 & 0 & 0
\end{array}\right)=\left(A_{1}, A_{2}\right) \\
& B=\left(\begin{array}{rrr}
1-1 & 0 \\
1 & 0 & 0 \\
\hline 1 & 0 & 0 \\
0 & -1 & 0 \\
1 & 1 & 1
\end{array}\right)=\left(\begin{array}{l}
B_{1} \\
B_{2}
\end{array}\right)
\end{aligned}
$$

Then

$$
A B=A_{1} B_{1}+A_{2} B_{2}=\left(\begin{array}{rrr}
0 & -1 & 0 \\
1 & -1 & 0 \\
1 & 1 & 0
\end{array}\right), \quad U=\left(\begin{array}{rrr}
0 & -1 & 0 \\
1 & 0 & 0 \\
0 & 1 & 0
\end{array}\right)
$$

and

$$
V=\left(\begin{array}{rrr}
0 & 0 & 0 \\
0 & -1 & 0 \\
1 & 0 & 0
\end{array}\right)
$$

Hence

$$
U^{*} V \neq 0_{3 \times 3} \quad \text { and } \quad V^{*} U=\left(\begin{array}{rrr}
0 & 1 & 0 \\
-1 & 0 & 0 \\
0 & 0 & 0
\end{array}\right)
$$

Thus $U^{*} V+V^{*} U=0$, which implies $E=0$. Now clearly $(U+V) E=0$, and Corollary 1.1 is applicable.

\section{Acknowledgement}

The authors would like to express their appreciation for helpful comments from two anonymous referees.

\section{References}

R. E. Cline (1965), 'Representation of the generalized inverse of sums of matrices', SIAM J. Numer. Anal., Ser. B, 2, 99-114. 
C. H. Hung and T. L. Markham (1975), 'The Moore-Penrose Inverse of a partitioned matrix $M=\left(\begin{array}{ll}A & D \\ B & C\end{array}\right)$, Linear Alg. and Appl. 11, 73-86.

W. C. Pye, T. L. Boullion, and T. A. Atchison (1973), 'The Pseudo-inverse of a Centrosymmetric Matrix', Linear Alg. and Appl. 6, 201-204.

Department of Mathematics,

Claflin College,

Orangeburg, South Carolina,

Columbia, S.C. 29208,

U.S.A.

Department of Mathematics,

University of South Carolina,

Columbia, S.C. 29208,

U.S.A. 